INPLASY

PROTOCOL

To cite: Wang et al. Prognositc value of serum bilirubin in patients with heart failure: protocol for a systematic review and meta-analysis. Inplasy protocol 202140116. doi:

10.37766/inplasy2021.4.0116

Received: 22 April 2021

Published: 23 April 2021

Corresponding author:

Huan Wang

670268513@qq.com

Author Affiliation:

Department of Cardiology, Guang'anmen Hospital, China Academy of Chinese Medical Sciences

Support: No.

Review Stage at time of this submission: The review has not yet started.

Conflicts of interest:

None declared.

\section{Prognositc value of serum bilirubin in patients with heart failure: protocol for a systematic review and meta- analysis}

\author{
Wang, $\mathrm{H}^{1}$; Jia, $\mathrm{Q}^{2}$; Shi, J3; Hu, Y4.
}

Review question / Objective: Heart failure (HF) is a critical disease, and increasing bilirubin is often accompanied by the development of HF, but the relationship between bilirubin and the prognosis of patients with heart failure is still unclear, so we implemented the study to explore the relationship between the two ones.

Condition being studied: HF is a complex clinical syndrome. The pathology involves in multiple organs and pathological segments. Therefore, its prognostic factors are very complicated. The EVREST study has revealed that abnormal liver function including total bilirubin can predict cardiovascular death. Large-scale CHARM studies have also confirmed that elevated total bilirubin is the strongest predictor of all-cause death, cardiovascular death and admissions in HF.

INPLASY registration number: This protocol was registered with the International Platform of Registered Systematic Review and Meta-Analysis Protocols (INPLASY) on 23 April 2021 and was last updated on 23 April 2021 (registration number INPLASY202140116).

\section{INTRODUCTION}

Review question / Objective: Heart failure (HF) is a critical disease, and increasing bilirubin is often accompanied by the development of $\mathrm{HF}$, but the relationship between bilirubin and the prognosis of patients with heart failure is still unclear, so we implemented the study to explore the relationship between the two ones. 
Condition being studied: HF is a complex clinical syndrome. The pathology involves in multiple organs and pathological segments. Therefore, its prognostic factors are very complicated. The EVREST study has revealed that abnormal liver function including total bilirubin can predict cardiovascular death. Large-scale CHARM studies have also confirmed that elevated total bilirubin is the strongest predictor of all-cause death, cardiovascular death and admissions in HF.

\section{METHODS}

Participant or population: Patients with heart failure.

Intervention: No.

\section{Comparator: No.}

Study designs to be included: Prospective study.

Eligibility criteria: (1)The meta-analysis will focus on prospective trials and retrospective studies to assess the prognostic value of bilirubin in patients with HF. (2) Participants who had reached the age of 18 and were diagnosed with heart failure were the eligible objects in the study. (3) The study of bilirubin as a prognostic factor will be included in this protocol, and we will also accept every evaluation of bilirubin provided by the study author. (4) The main results will include: MACE and all-cause mortality.Secondary results will include: Length of stay in intensive/cardiovascular intensive care unit; Use of vasoactive drugs.

Information sources: Medline, Cochrane Library, Embase, Web of Science, Cochrane Clinical Trials Database.

Main outcome(s): (1)MACE, including cardiovascular events, myocardial infarction, and stroke; (2)all-cause mortality. All the results consist of inhospital and long-term follow-up events/ mortality.
Quality assessment / Risk of bias analysis: Cochrane Collaborations 'risk of bias' tool will be used to fairly review the risk of bias identified in this study.

Strategy of data synthesis: The data were analysed by Revman 5.3.5 software and STATA 14.0 software.

Subgroup analysis: If necessary, we will analysis gender, age, BNP/NT-proBNP level, follow-up time to evaluate the influence on prognostic indicators.

Sensitivity analysis: Sensitivity check will be conducted to ensure the robustness and exclude studies with poor quality or high risk of bias or unclear methodological data.

\section{Country(ies) involved: China.}

Keywords: bilirubin; Heart failure; Prognosis.

Contributions of each author:

Author 1 - Huan Wang.

Author 2 - Qiulei Jia.

Author 3 - Jingjing Shi.

Author 4 - Yuanhui Hu. 